\title{
Both deterioration and improvement in a
of daily living are related to falls: a 6-year follow-up of the general elderly population study Good Aging in Skåne
}

\author{
This article was published in the following Dove Press journal: \\ Clinical Interventions in Aging \\ 28 October 2014 \\ Number of times this article has been viewed
}

\section{Magnus Stenhagen \\ Henrik Ekström \\ Eva Nordell \\ Sölve Elmståhl}

Department of Health Sciences, Division of Geriatric Medicine, Lund University, Skåne University Hospital, Malmö, Sweden
Correspondence: Magnus Stenhagen Division of Geriatric Medicine, Skåne University Hospital, CRC, Building 28, Floor 13, Jan Waldenströms gata 35, SE-205 02 Malmö, Sweden Email magnus.stenhagen@med.lu.se
Objectives: To determine the relationship between long-term change in activities of daily living (ADL) and falls in the elderly and to identify characteristics of groups at risk for falls.

Methods: This was a 6-year, prospective cohort study using data from the Good Aging in Skåne study in southern Sweden, involving 1,540 elderly subjects, including the oldest-old (age, 60-93 years). The subjects were recruited from the general population. ADL was measured at a baseline and follow-up assessment, using Sonn and Åsberg's revised scale and the ADL staircase. Falls were recorded in a period of 6 months before the follow-up assessment. The association between falls and change in ADL was calculated using adjusted, multiple logistic regression analysis and presented in odds ratios (ORs).

Results: Thirteen percent of the study population reported one or several falls in the measured period. Over the course of 6 years, one in four participants changed their ADL status, and parts of this category had an increased risk for falls compared with those who stayed independent in ADL or who had no change in the ADL staircase. Groups with different characteristics had a prominent risk for falls: those with a reduction of two to eight steps in the ADL staircase (OR, 4.05; 95\% confidence interval [CI], 1.62-10.11) and those becoming independent from dependency in instrumental ADL (OR, 4.13; 95\% CI, 1.89-9.00). The former group had advanced age with a greater burden of cognitive impairment, gait disability, arrhythmia, and fall risk medications. The latter group had a higher prevalence of ischemic heart disease and low walking speed.

Conclusion: Both deterioration and improvement in ADL over the course of 6 years increased the risk for falls in a general elderly population. Interventional efforts may require different strategies, as groups with different characteristics were at risk. Those at risk with improved ADL function may have a history of sufficient burden of comorbidity combined with obtained mobility for exposure to a fall event.

Keywords: accidental falls, activities of daily living, prospective, elderly, general population

\section{Introduction}

In a "graying world" with increased longevity, falls in the elderly stand for substantial morbidity and mortality. ${ }^{1-3}$ Without preventive and interventional efforts, falls and associated injuries may increase substantially in the coming decades, as they seem to increase exponentially with advancing age. ${ }^{4,5}$ In Sweden, 875 individuals died directly from falls in 2011 compared with 325 individuals who died in road traffic accidents. ${ }^{6}$ In addition to fatal and severe injuries such as hip fractures and head injuries, the consequences of falls are broad and contribute to immobility and 
premature institutionalization. ${ }^{1,7,8}$ Prospective cohort studies indicate that falls seem to be an independent determinant for functional decline and dependency in activities of daily living (ADL) in a general elderly population. ${ }^{9-11}$ In addition, a faller can develop a "fear of falling," conceptualized as a loss of self-confidence, self-efficacy, and activity avoidance. ${ }^{12,13}$ Altogether, falls are a major health burden, meeting the criteria for preventive measures. ${ }^{11}$ To optimize preventive interventions, there is a need for epidemiological studies to target individuals at risk. ${ }^{14,15}$

According to a joint American and British guideline, several epidemiological studies have identified numerous risk factors for falls in a general elderly population; thus, it is well established that causes of falls are multifactorial. ${ }^{1}$ In addition to distinct risk factors such as advanced age and general frailty, several factors and markers seem to be associated with mobility and the musculoskeletal system (eg, muscle weakness, gait deficit, and the use of a walking aid). ${ }^{16-19}$ Furthermore, individuals with low overall function with impairment or dependency in ADL make up a group at risk. 1,7,20

In geriatric medicine, clinicians have long recognized the importance of diagnosing a disease combined with an assessment of its sequelae on overall function. The concept of comprehensive geriatric assessment has been used in this context. ${ }^{21}$ From the perspective of the affected individual (eg, after a stroke), the most central aspect of quality of life may be the ability to remain independent in the home environment. ${ }^{22-24}$ The concept of ADL has been developed and refined since the 1950 s and is a cornerstone of geriatric rehabilitation. ${ }^{25}$ It is useful in assessing the individual need for care and the progress of rehabilitation. In an epidemiological perspective, it can be used to evaluate the longitudinal progress of disability in the aging process. ${ }^{22,26}$ As a result of a growing elderly population, an increasing proportion of individuals with functional disability and loss of independence is expected. ${ }^{24,27}$ With an impending parallel increase of falls in the elderly, it is relevant to study the interaction between longitudinal change in ADL and falls. There is, to our knowledge, no previous study that analyzes the association between long-term change in ADL function and the risk for falls in a general elderly population. This aspect can be useful in identifying groups at risk and in better understanding the natural course of disability over time.

The aim of this study was to determine the relation between change in ADL and falls over the course of 6 years in a general elderly population and to identify characteristics of groups at risk for falls.

\section{Methods Study population}

This was a 6-year, prospective cohort study based on data from the ongoing, longitudinal population study Good Aging in Skåne, which is part of the Swedish National Study of Aging and Care. ${ }^{28,29}$ Participants from five municipalities, both urban and rural, in the region of Skåne in southern Sweden, were randomly selected using the National Population Register. The only exclusion criterion was the inability to speak and understand Swedish. Both men and women from the nine age cohorts $(60,66,72,78,81,84,87,90$, and 93 years) were invited. The response rate was $60 \%$. Initially, 2,931 subjects participated in a baseline assessment from February 2001 to July 2004. The assessment was performed at an outpatient research center or at the subject's own home or in sheltered housing. All subjects were invited to a 6-year follow-up assessment taking place between March 2007 and December 2011. The response rate of those not deceased or vacated was $80 \%$, with 1,831 subjects participating. Fallers at baseline and those with missing data on falls and ADL were excluded from the study ( $n=133 / 158)$. Finally, 1,540 subjects met the criteria for inclusion in the study population.

Data were solely recorded at the baseline and at the 6-year follow-up assessment. The incidence of falls in the period of 6 months before the follow-up was recorded by a face-to-face interview with a physician. Using a structured questionnaire, the subject was asked, "Have you fallen once or more in the last 6 months?" Those who had fallen once or more were dichotomized as fallers. Because of the extensive scope of the study, no continuous assessment of the incidence of falls could be carried out throughout the study.

Sociodemographic factors and assessment of physical activity were obtained using self-reported questionnaires at baseline. Low physical activity was classified as scoring 1 or 2 points on the 6-level physical activity scale by MattiassonNilo et al. ${ }^{30}$ The need for a walking device was assessed by a registered nurse. Walking speed was measured by timing the subject's maximal walking speed over $15 \mathrm{~m}$ without running. Low walking speed was defined as obtaining a time above the median value of the study population.

\section{Comorbidity and medications}

A detailed medical examination, including medical history and records, was made by a physician at the baseline and follow-up assessments. The classification of medical diagnoses was based on the International Classification of Diseases, Tenth Revision, at the baseline assessment. ${ }^{31}$ The factor heart disease was composed of angina, myocardial infarction, and 
arrhythmia. Heart failure with symptoms was based on a score of 2-4 in the New York Heart Association classification of congestive heart failure. ${ }^{32}$ Cognitive function was screened using the Mini-Mental State Examination scale. ${ }^{33}$ A score below 24 points on that scale defined suspected cognitive impairment. The use of medications was registered according to the Anatomical Therapeutic Chemical classification system at the baseline assessment. ${ }^{34}$ The use of fall risk drugs included the use of sedatives/hypnotics, neuroleptics, anticholinergic drugs, antihypertensives (beta-blockers, angiotensin-converting enzyme inhibitors, calcium channel blockers), and/or diuretics (thiazide- and loop-diuretics).

\section{ADL assessment}

ADL was assessed through self-report according to Sonn and Åsberg's revised ADL scale, using identical protocols at the baseline and follow-up assessment. This instrument is a development of the Katz ADL index and has been tested for both reliability and validity. ${ }^{35,36}$ The instrument includes ten activities, which can be divided into four instrumental activities of daily living (iADL: cleaning, grocery shopping, transportation, and cooking), and six personal activities (pADL: bathing, dressing and undressing, toileting, mobility, incontinence, and food intake). These activities can be arranged hierarchically in the form of the ordinal, 10-step ADL staircase, where a higher score indicates a lower functional ability. Those dependent in activities with a higher score (eg, food intake) are estimated to be dependent in activities with a lower score.

Dependency in $\mathrm{AADL}$ was defined as independence in the activities of pADL while being dependent in at least one activity in iADL. Dependency in pADL was defined as being dependent in at least one activity in pADL.

\section{Statistical methods}

The study population was divided into eight groups according to their change or no change in ADL status from baseline to the follow-up assessment ( $n=1,540$; Table 1). Furthermore, the individual change in the ADL staircase was calculated by subtracting the baseline score from the score at the follow-up assessment. Using this method, a positive difference corresponded to a functional decline. The size of the analyzed group in this measurement was reduced, with 340 subjects missing as a result of the sensitivity of the missing data in computing the score of the ADL staircase. Those with complete data were divided into four groups according to their difference or no difference in score over time $(n=1,200$; Table 1$)$.

The association between each individual group and the incidence of one or more falls 6 months before the follow-up assessment was initially analyzed using an unadjusted logistic regression analysis, with the results presented in crude odds ratios (Table 1). In the analyses of the groups with change in ADL status, the group who stayed independent in ADL was used as the reference $(n=831)$. In the corresponding analyses

Table I Change in activities of daily living (ADL) and the ADL staircase ( 0 to 10 steps) over the course of 6 years and association to falls at the follow-up assessment

\begin{tabular}{|c|c|c|c|c|}
\hline Status in ADL & $\mathbf{n}$ & $\begin{array}{l}\text { Crude odds ratio, } \\
\text { (95\% confidence interval) }\end{array}$ & $\begin{array}{l}\text { Adjusted odds ratio } \\
\text { ( } 95 \% \text { confidence interval)* }\end{array}$ & $\begin{array}{l}\text { Adjusted odds ratio } \\
(95 \% \text { confidence interval })^{\dagger}\end{array}$ \\
\hline \multicolumn{5}{|l|}{ No change in ADL } \\
\hline Stayed dependent in ADL & 295 & $3.16(2.18-4.59)$ & $2.5 \mathrm{I}(1.64-3.84)$ & $2.42(1.56-3.76)$ \\
\hline Stayed independent in ADL (reference) & 831 & I & 1 & 1 \\
\hline \multicolumn{5}{|l|}{ Change in ADL } \\
\hline Became dependent in ADL & 266 & $2.64(1.78-3.92)$ & $2.02(1.30-3.14)$ & $1.97(1.25-3.10)$ \\
\hline Toward instrumental ADL dependency & 85 & $1.87(0.97-3.63)$ & $\mathrm{I} .80(0.85-3.8 \mathrm{I})$ & $1.58(0.72-3.48)$ \\
\hline Toward personal ADL dependency & 181 & $3.03(1.96-4.69)$ & $2.26(1.40-3.63)$ & $2.31(1.42-3.76)$ \\
\hline Became independent in ADL & 148 & $2.32(I .4 I-3.8 I)$ & $2.20(1.38-3.64)$ & $2.25(1.35-3.75)$ \\
\hline From instrumental ADL dependency & 44 & $3.35(1.59-7.09)$ & $3.69(1.73-7.87)$ & $4.13(1.89-9.00)$ \\
\hline From personal ADL dependency & 104 & $1.92(I .05-3.5 I)$ & $1.69(0.91-3.1 \mathrm{I})$ & $1.66(0.89-3.10)$ \\
\hline \multicolumn{5}{|l|}{ Change in ADL staircase } \\
\hline Two to eight step reduction & 29 & $5.43(2.44-12.09)$ & $4.48(1.87-10.77)$ & $4.05(1.62-10.11)$ \\
\hline One step reduction & 166 & I.4I (0.84-2.38) & $1.29(0.75-2.20)$ & $1.25(0.72-2.15)$ \\
\hline Zero (reference) & 883 & 1 & 1 & 1 \\
\hline One to three step improvement & 122 & $2.02(1.19-3.45)$ & $\mathrm{I} .87(\mathrm{I} .09-3.2 \mathrm{I})$ & $1.91(1.11-3.29)$ \\
\hline
\end{tabular}

Notes: Risk for falls calculated with unadjusted logistic regression and adjusted multiple logistic regression analysis. *Adjusted for age and sex. ${ }^{\dagger} A d j u s t e d$ for age, sex, heart disease, heart failure with symptoms, and use of fall risk drugs.

Abbreviations: ADL, activities of daily living; n, number. 
of change in the ADL staircase, the reference group included those with no change in score $(n=883)$.

To adjust for confounding factors, a multiple logistic regression analysis was used, adjusted for age, sex, and comorbidity. This model was based on a preceding prospective analysis of the study population, ${ }^{20}$ in which a principal component analysis revealed three independent, thematic components predicting falls: reduced mobility (including indoor adaptation, osteoarthritis of hip and/or knee, low walking speed, and impaired mobility), functional impairment (including dependence in pADL and nocturia), and heart dysfunction (including heart disease, heart failure with symptoms, and the use of fall risk drugs). As the two former components were assumed to be intimately associated with ADL, they were not used in the multiple regression model of this study in an attempt to reduce multicollinearity between the explanatory variables for falls. The independent component heart dysfunction, including the factors heart disease, heart failure with symptoms, and use of fall risk drugs, was used in the final model to adjust for comorbidity. The results of these analyses were presented in odds ratios (ORs) (Table 1).

Finally, a descriptive analysis was made of the characteristics of selected groups with risk for falls (Table 2). The proportions of a number of categories in each group were compared with those who stayed independent in ADL $(n=831)$. The statistical differences were analyzed using Pearson's chi-squared test complemented with Fisher's exact test when the sample sizes were small.

SPSS software package version 20 was used for all statistical analyses. Throughout the study, a 95\% confidence interval $(\mathrm{CI})$ and a $P$-value $<0.05$ defined significance. All $P$-values were two-sided.

\section{Ethics approval}

The study was approved by the Ethical Committee of Lund University, Sweden, and all participants gave their written informed consent.

\section{Results}

The study population consisted of $46 \%$ men and $54 \%$ women. The age range was 60-93 years at the baseline assessment, with a mean age of 68.8 (standard deviation, 8.8) years. Sixtyfour percent of the participants were in their sixties, and $20 \%$ were in their seventies. Fourteen percent were in their eighties, and 3\% were older than 90 years. A majority lived in an urban setting (91\%); 9\% lived in a rural environment. More than one in three lived alone (37\%), and 11 subjects were recruited from sheltered housing. Twenty-two percent had a higher education, and 10\% were born outside Sweden. Six percent of the study population had suspected cognitive impairment. Thirteen percent reported one or several falls in the period of 6 months before the 6-year follow-up assessment.

Slightly more than one in four participants changed their status in ADL over the course of 6 years (Table 1). Approximately half the population remained independent in ADL, and almost one in five remained dependent in ADL. Seventeen percent went from independence in ADL to dependence in pADL or iADL. Almost one in ten went from dependence in $\mathrm{pADL}$ or $\mathrm{AADL}$ to independence in ADL. In those with complete data on the ADL staircase, almost three in four had no change in their score. Slightly more than $16 \%$ increased their score, indicating a functional decline, whereas almost $8 \%$ decreased their score.

In the adjusted multiple regression analysis, losing independency in ADL increased the risk for falls (Table 1; OR, 1.97; 95\% CI, 1.25-3.10), especially in those becoming dependent in pADL (OR, 2.31; 95\% CI, 1.42-3.76). The group with a functional decline of two to eight steps in the ADL staircase had a prominent risk for falls (OR, 4.05; 95\% CI, 1.62-10.11). Those becoming independent in ADL had an increased risk for falls (OR, 2.25; 95\% CI, 1.35-3.75), especially those becoming independent from dependency in iADL, displaying an OR of 4.13 (95\% CI, 1.89-9.00). In line with this, an improvement of one to three steps in the ADL staircase increased the risk for falls (OR, 1.91; 95\% CI, 1.11-3.29). The overall association between change in the ADL staircase and the risk for falls in ORs is graphically presented in Figure 1.

The descriptive analysis of the selected groups with increased risk for falls revealed different characteristics of the subjects at risk. Those with a decline of two to eight steps in the ADL staircase were significantly older with a lower walking speed and higher use of a walking device compared with those staying independent in ADL (Table 2). This group had a higher prevalence of arrhythmia and suspected cognitive impairment, with a greater consumption of sedative/ hypnotic and diuretic drugs. Those becoming independent from iADL dependency had a significant lower walking speed and higher prevalence of ischemic heart disease (angina and myocardial infarction).

\section{Discussion \\ Main findings and clinical implications}

According to results of this study, $17 \%$ of the study population became dependent in ADL and $16 \%$ had a functional decline in the ADL staircase over 6 years. These numbers 


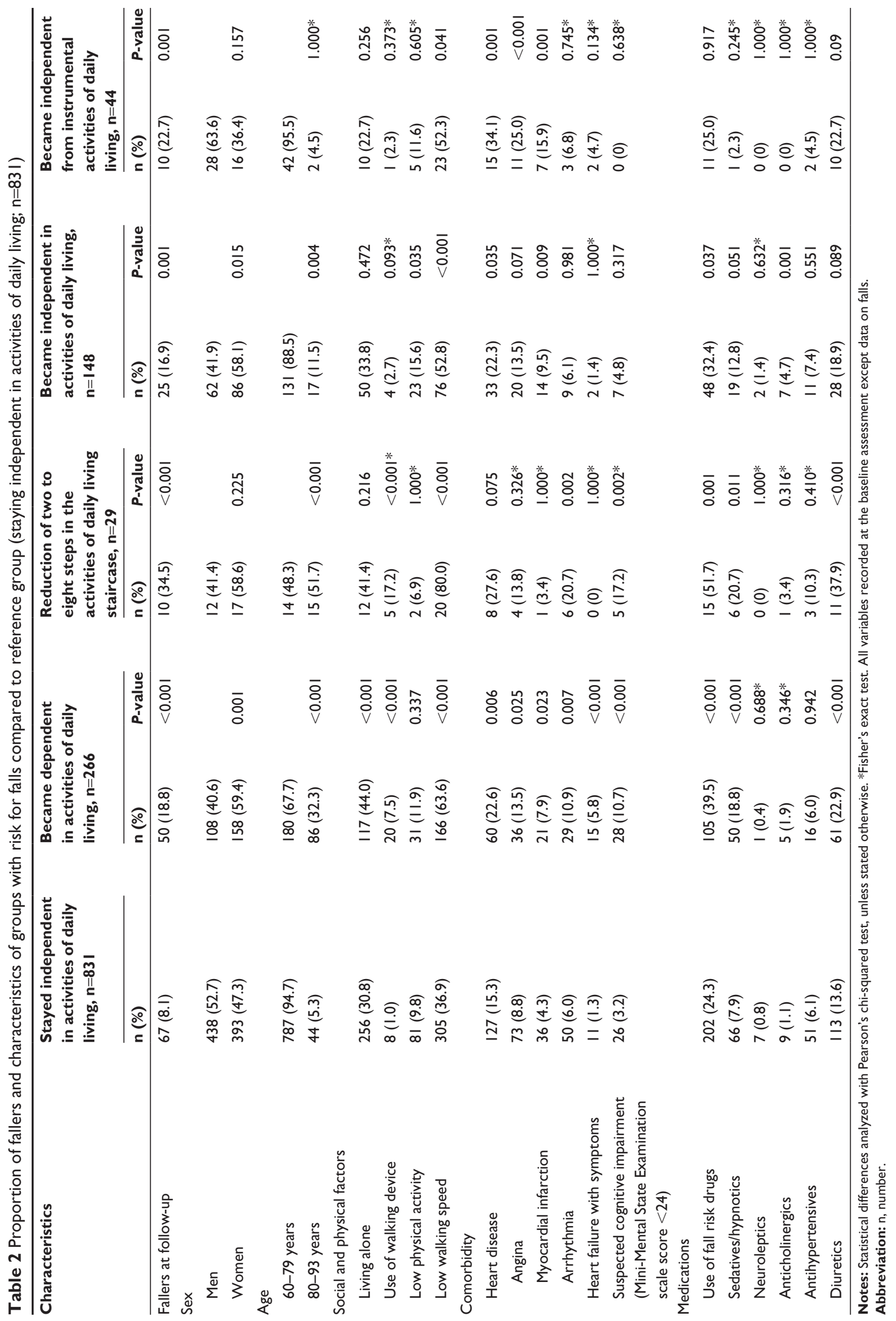




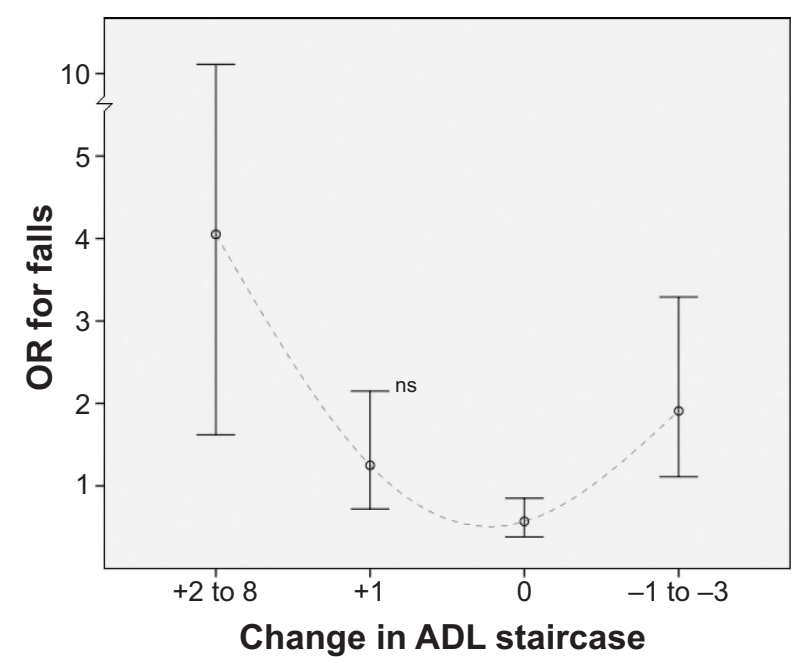

Figure I Change in the activities of daily living (ADL) staircase (0-10 steps) over the course of 6 years and association to falls at the follow-up assessment.

Notes: An increased score corresponds to a functional decline. Risk presented in odds ratios (ORs) with $95 \%$ confidence interval as whiskers $(n=1,200)$.

Abbreviations: ns, not significant; $n$, number.

correspond well with a previous prospective cohort study of the elderly in which $19 \%$ became more dependent in functional ability over the course of 6 years. ${ }^{37}$ In total, one-quarter of the study population changed their ADL status over the course of 6 years. This category included the groups with the most prominent risk for falls; for example, the group with a marked decline in the ADL staircase (two to eight steps). This seems to be a group with impairments in multiple domains, which can generate frailty with a reduced compensatory ability. This group fulfilled many of the criteria of a "geriatric syndrome," with a higher prevalence of advanced age, female sex, gait disability, arrhythmia, cognitive impairment, and consumption of fall risk medications.

Somewhat paradoxically, those who improved their functional ability and became independent from ADL dependency had an increased risk for falls. Hence, the risk for falls in relation to change in ADL could be interpreted as a U-shaped curve (Figure 1). According to reviews, this pattern has been described in the literature regarding the association between physical ability and falls in the elderly, where the risk for falls is suggested to be generally reduced among physically active people, with a potential increased risk among the most active and inactive individuals. ${ }^{18,38}$ In a large, prospective cohort study of a general elderly population, the most active quartile had a significantly greater fall risk than the least active quartile. ${ }^{39}$

The group that became independent from iADL dependency had the most marked risk for falling in our study, although the confidence interval was wide. This group was seemingly younger, physically and cognitively intact, with moderate consumption of risk medications, but with a higher prevalence of ischemic heart disease. A majority of this group was men, although the sex difference was not statistically significant $(P=0.157)$. In a study of 9,250 elderly individuals from a general population, the risk for falls peaked at an intermediate ADL limitation stage, an assumed point at which the individual had significant disabilities but sufficient function to remain partially active..$^{40}$ In line with this, this particular group at risk in this study may be subjects with a sufficient burden of comorbidity combined with obtained mobility for exposure to a fall event.

Both groups with prominent risk for falls had an increased prevalence of heart dysfunction (arrhythmia and ischemic heart disease, respectively). This is notable, as the prevalence of cardiovascular causes of falls in the general population is largely unknown and there may be overlap between the symptoms of falls and syncope in the elderly. ${ }^{1}$ Those who became independent from dependency in iADL had an increased prevalence of angina and myocardial infarction. As the presence of this condition was assessed at the baseline, one can hypothesize that these individuals were recommended to improve their activity by medical providers after their diagnosis. More research is needed on the associations among falls, ischemic heart disease, and improvement in ADL, as they may be of significant clinical relevance for practitioners who diagnose and treat heart conditions in this age group.

The overall finding of this study implies there are groups of different characteristics at risk for falls in a general elderly population. Therefore, interventional efforts may require different strategies. A meta-analysis of six studies reported that physical training reduced the number of individuals who fell among older adults. ${ }^{41}$ Contradictorily, according to a literature review, it seemed questionable whether any intervention program with physical activity could reduce the number of falls among elderly individuals living in institutions ${ }^{42}$ This intervention may be too risky in this particular group, leading to falls during exercise.

\section{Strengths and limitations}

This study used Sonn and Åsberg's revised ADL scale, an assessment developed from the Katz ADL index. This instrument is internationally established, previously validated, and tested for reliability. ${ }^{35,36}$ Furthermore, the study population consisted of a rather large sample of elderly men and women, including the oldest-old, randomly selected from a general population. This methodological approach improves the generalizability of the results. Furthermore, 
this study investigated the change in ADL function over a rather lengthy period of time. The results showed that ADL function is a dynamic process, and a significant part of the study population altered their ADL status over the course of 6 years. The groups with the most prominent risk for falls had a change in their ADL function. This may underline the importance of studies analyzing functional assessment over time to identify groups at risk for falls and to gain understanding of the progress of disability and rehabilitation in the elderly.

The response rate at the follow-up assessment was $80 \%$. Despite this, an attrition bias cannot be ruled out. In a previous attrition analysis of the studied cohort, the nonparticipants were significantly older with a greater burden of somatic disease, cognitive impairment, and dependency in ADL. ${ }^{20}$ This misrepresentation of seemingly more healthy subjects may have diluted the overall results in our study. Unfortunately, the internal attrition of the ADL data was not insignificant, as 340 subjects did not have complete data in the ADL staircase. You can hypothesize that the older, frailer, and more cognitively impaired did not complete the full assessment to the same extent as the healthier subjects, excluding subjects with potential ADL deterioration. Despite this, no intent to impute data was made to reduce possible misclassification.

Because of the scope of the study, we were not able to monitor falls and other adverse events continuously from the baseline to the follow-up assessment. Although the study was prospective in its design, data on falls were recorded retrospectively 6 months before the follow-up assessment. This methodological limitation in recording the outcome variable could affect the temporality and causal interpretation of the results. Furthermore, in using a dichotomized approach, the absolute frequency and severity of the falls were not accounted for. This can be seen as a limitation, as the outcomes of a fall event in the elderly can be diverse. Despite this, a dichotomized outcome variable could be adequate, as any fall can be considered a potentially serious event for the older individual, in whom even low-impact falls can have severe consequences. ${ }^{43}$

\section{Conclusion}

One in four participants changed their ADL status over the course of 6 years in a general elderly population. Parts of this category had a higher risk for falls compared with those who stayed independent or had no change in the ADL staircase. Fall-preventive interventions may require different strategies, as groups with different characteristics and both deterioration and improvement in ADL were at prominent risk, including those with a marked decline in ADL, an elderly group with a greater burden of cognitive impairment, gait disability, arrhythmia, and consumption of sedatives/ hypnotics and diuretics, and those becoming independent from iADL dependency, a seemingly younger group with higher prevalence of ischemic heart disease. This group may have a history of sufficient comorbidity combined with obtained mobility for exposure to a fall event.

\section{Acknowledgments}

The project Good Aging in Skåne, part of the Swedish National Study on Aging and Care, was supported by the Swedish Ministry of Health and Social Affairs, the county Region Skåne, the Medical Faculty at Lund University, and the Vårdal Institute.

\section{Disclosure}

The authors report no conflicts of interest in this work.

\section{References}

1. Guideline for the prevention of falls in older persons. American Geriatrics Society, British Geriatrics Society, and American Academy of Orthopaedic Surgeons Panel on Falls Prevention. J Am Geriatr Soc. 2001;49(5):664-672.

2. Kannus P, Sievänen H, Palvanen M, Järvinen T, Parkkari J. Prevention of falls and consequent injuries in elderly people. Lancet. 2005;366(9500):1885-1893.

3. Kalache A, Keller I. The greying world: a challenge for the twenty-first century. Sci Prog. 2000;83(Pt 1):33-54.

4. World Health Organization. Global report on falls prevention in older age. Geneva: WHO; 2007.

5. Kannus P, Parkkari J, Koskinen S, et al. Fall-induced injuries and deaths among older adults. JAMA. 1999;281(20):1895-1899.

6. The National Board of Health and Welfare. Causes of Death in Sweden 2011. Availabe from: http://www.socialstyrelsen.se/Lists/Artikelkata$\log$ /Attachments/19001/2013-2-30.pdf. Accessed January 15, 2014. Swedish.

7. Rubenstein LZ. Falls in older people: epidemiology, risk factors and strategies for prevention. Age Ageing. 2006;35(suppl 2):ii37-ii41.

8. Tinetti ME, Williams CS. Falls, injuries due to falls, and the risk of admission to a nursing home. N Engl J Med. 1997;337(18):1279-1284.

9. Sekaran NK, Choi H, Hayward RA, Langa KM. Fall-associated difficulty with activities of daily living in functionally independent individuals aged 65 to 69 in the United States: a cohort study. $J$ Am Geriatr Soc. 2013;61(1):96-100.

10. Stel VS, Smit JH, Pluijm SMF, Lips P. Consequences of falling in older men and women and risk factors for health service use and functional decline. Age Ageing. 2004;33(1):58-65.

11. Tinetti ME, Williams CS. The effect of falls and fall injuries on functioning in community-dwelling older persons. J Gerontol A Biol Sci Med Sci. 1998;53(2):M112-M119.

12. Jørstad EC, Hauer K, Becker C, Lamb SE; ProFaNE Group. Measuring the psychological outcomes of falling: a systematic review. $J$ Am Geriatr Soc. 2005;53(3):501-510.

13. Scheffer AC, Schuurmans MJ, van Dijk N, van der Hooft T, de Rooij SE. Fear of falling: measurement strategy, prevalence, risk factors and consequences among older persons. Age Ageing. 2008;37(1):19-24. 
14. Karlsson MK, Ribom E, Nilsson J-Å, et al. Inferior physical performance tests in 10,998 men in the MrOS study is associated with recurrent falls. Age Ageing. 2012;41(6):740-746.

15. Gillespie LD, Robertson MC, Gillespie WJ, et al. Interventions for preventing falls in older people living in the community. Cochrane Database Syst Rev. 2012;9:CD007146.

16. Ganz DA, Bao Y, Shekelle PG, Rubenstein LZ. Will my patient fall? JAMA. 2007;297(1):77-86.

17. O'Loughlin JL, Robitaille Y, Boivin JF, Suissa S. Incidence of and risk factors for falls and injurious falls among the community-dwelling elderly. Am J Epidemiol. 1993;137(3):342-354.

18. Bergland A. Fall risk factors in community-dwelling people. Nor J Epidemiol. 2012;22:151-164.

19. Deandrea S, Lucenteforte E, Bravi F, Foschi R, La Vecchia C, Negri E. Risk factors for falls in community-dwelling older people: a systematic review and meta-analysis. Epidemiology. 2010;21(5):658-668.

20. Stenhagen M, Ekström H, Nordell E, Elmståhl S. Falls in the general elderly population: a 3- and 6-year prospective study of risk factors using data from the longitudinal population study 'Good ageing in Skane'. BMC Geriatr. 2013;13(81):81.

21. Stuck AE, Siu AL, Wieland GD, Adams J, Rubenstein LZ. Comprehensive geriatric assessment: a meta-analysis of controlled trials. Lancet. 1993;342(8878):1032-1036.

22. Guralnik JM, Branch LG, Cummings SR, Curb JD. Physical performance measures in aging research. J Gerontol. 1989;44(5):M141-M146.

23. Balzi D, Lauretani F, Barchielli A, et al. Risk factors for disability in older persons over 3-year follow-up. Age Ageing. 2010;39(1):92-98.

24. Wang L, van Belle G, Kukull WB, Larson EB. Predictors of functional change: a longitudinal study of nondemented people aged 65 and older. $J$ Am Geriatr Soc. 2002;50(9):1525-1534.

25. Katz S, Ford AB, Moskowitz RW, Jackson BA, Jaffe MW. Studies of Illness in the Aged. The Index of ADL: A Standardized Measure of Biological and Psychosocial Function. JAMA. 1963;185(12): 914-919.

26. Katz S, Downs TD, Cash HR, Grotz RC. Progress in development of the index of ADL. Gerontologist. 1970;10(1):20-30.

27. Stuck AE, Walthert JM, Nikolaus T, Büla CJ, Hohmann C, Beck JC. Risk factors for functional status decline in community-living elderly people: a systematic literature review. Soc Sci Med. 1999;48(4):445-469.

28. Ekström H, Elmståhl S. Pain and fractures are independently related to lower walking speed and grip strength: results from the population study “Good Ageing in Skåne”. Acta Orthop. 2006;77(6):902-911.

29. Lagergren M, Fratiglioni L, Hallberg IR, et al; The Swedish National Study on Aging and Care (SNAC). A longitudinal study integrating population, care and social services data. The Swedish National study on Aging and Care (SNAC). Aging Clin Exp Res. 2004;16(2):158-168.
30. Mattiasson-Nilo I, Sonn U, Johannesson K, Gosman-Hedström G, Persson GB, Grimby G. Domestic activities and walking in the elderly: evaluation from a 30-hour heart rate recording. Aging (Milano). 1990;2(2):191-198.

31. International Statistical Classification of Diseases and Related Health Problems,10th Revision [webpage on the Internet]. Geneva: World Health Organization. Available from: http://apps.who.int/classifications/ icd10/browse/2010/en. Accessed January 15, 2014.

32. American Heart Association. Classes of Heart Failure. Available from: http://www.heart.org/HEARTORG/Conditions/HeartFailure/ AboutHeartFailure/Classes-of-Heart-Failure_UCM_306328_Article. jsp. Accessed January 15, 2014

33. Folstein MF, Folstein SE, McHugh PR. "Mini-mental state". A practical method for grading the cognitive state of patients for the clinician. J Psychiatr Res. 1975;12(3):189-198.

34. WHO Collaborating Centre for Drug Statistics Methodology. International language for drug utilization research. Available from: http:// www.whocc.no/. Accessed January 15, 2014.

35. Asberg KH, Sonn U. The cumulative structure of personal and instrumental ADL. A study of elderly people in a health service district. Scand J Rehabil Med. 1989;21(4):171-177.

36. Sonn U, Asberg KH. Assessment of activities of daily living in the elderly. A study of a population of 76-year-olds in Gothenburg, Sweden. Scand J Rehabil Med. 1991;23(4):193-202.

37. Schroll M, Avlund K, Davidsen M. Predictors of five-year functional ability in a longitudinal survey of men and women aged 75 to 80. The 1914-population in Glostrup, Denmark. Aging (Milano). 1997;9(1-2):143-152.

38. Moayyeri A. The association between physical activity and osteoporotic fractures: a review of the evidence and implications for future research. Ann Epidemiol. 2008;18(11):827-835.

39. Chan BKS, Marshall LM, Winters KM, Faulkner KA, Schwartz AV, Orwoll ES. Incident fall risk and physical activity and physical performance among older men: the Osteoporotic Fractures in Men Study. Am J Epidemiol. 2007;165(6):696-703.

40. Henry-Sánchez JT, Kurichi JE, Xie D, Pan Q, Stineman MG. Do elderly people at more severe activity of daily living limitation stages fall more? Am J Phys Med Rehabil. 2012;91(7):601-610.

41. Gregg EW, Pereira MA, Caspersen CJ. Physical activity, falls, and fractures among older adults: a review of the epidemiologic evidence. J Am Geriatr Soc. 2000;48(8):883-893

42. Karlsson MK, Nordqvist A, Karlsson C. Physical activity, muscle function, falls and fractures. Food Nutr Res. 2008:52.

43. Sterling DA, O'Connor JA, Bonadies J. Geriatric falls: injury severity is high and disproportionate to mechanism. J Trauma. 2001;50(1):116-119.
Clinical Interventions in Aging

\section{Publish your work in this journal}

Clinical Interventions in Aging is an international, peer-reviewed journal focusing on evidence-based reports on the value or lack thereof of treatments intended to prevent or delay the onset of maladaptive correlates of aging in human beings. This journal is indexed on PubMed Central, MedLine,
Dovepress

CAS, Scopus and the Elsevier Bibliographic databases. The manuscript management system is completely online and includes a very quick and fair peer-review system, which is all easy to use. Visit http://www.dovepress. com/testimonials.php to read real quotes from published authors. 